\title{
Aerodynamic Analysis of a harmonically Morphing Flap Using a Hybrid Turbulence Model and Dynamic Meshing
}

\author{
Chawki Abdessemed ${ }^{1}$, Yufeng $\mathrm{Yao}^{2}$, Abdessalem Bouferrouk ${ }^{3}$, Pritesh Narayan ${ }^{4}$ \\ Department of Engineering Design and Mathematics, University of the West of England, Coldharbour Lane, \\ Frenchay, Bristol BS16 1QY, United Kingdom.
}

\begin{abstract}
A numerical study of the NACA 0012 airfoil fitted with a harmonically morphing trailing edge flap (TEF) is performed at an angle of attack of $4^{\circ}$ and a Reynolds number $R e=0.62 \times 10^{6}$. The study focuses on high frequency, low amplitude configurations for the morphing flap and their effects on the aerodynamic performance and flow structures in the wake. Dynamic meshing methods implemented in the commercial software Ansys Fluent and driven by an in-house user-defined function (UDF) were used to model the TEF deformation using a modified unsteady parametrization. The Stress Blended Eddy Simulation (SBES) hybrid turbulence model was used for all parametric studies. For a fixed amplitude, a range of morphing frequencies (lower and higher than the natural shedding frequency) was explored. Obtained results show that at certain high frequencies a slight increase in aerodynamic efficiency could be achieved compared with a baseline design. When the morphing frequency was fixed at its shedding value, the range of amplitudes investigated indicated the presence of an optimal morphing amplitude for which up to $3 \%$ increased aerodynamic efficiency could be obtained. Some preliminary results for upstroke and downstroke TEF oscillations are briefly presented to illustrate some differences compared with the main morphing strategy adopted in the paper.
\end{abstract}

\section{Nomenclature}

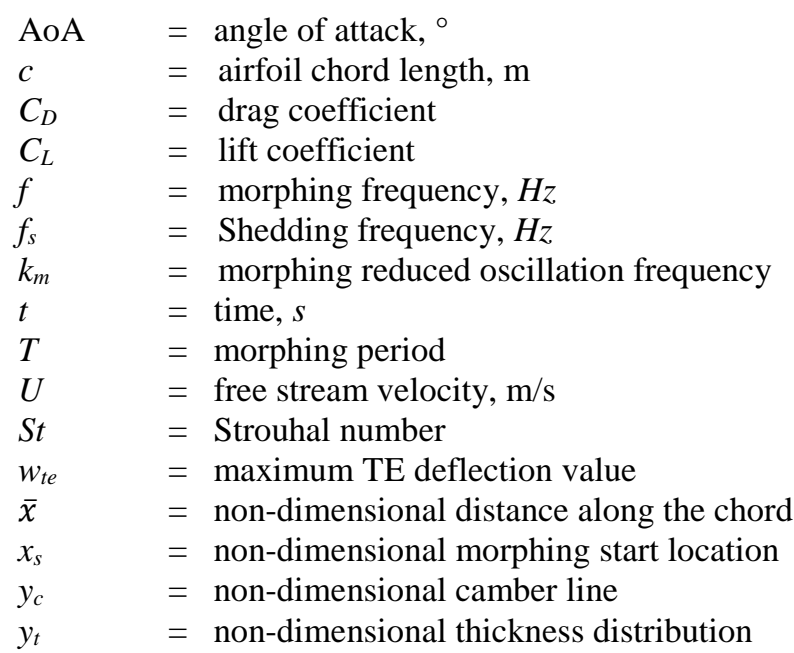

\footnotetext{
${ }^{1} \mathrm{PhD}$ student, Department of Engineering Design and Mathematics, AIAA Student member.

${ }^{2}$ Professor, Department of Engineering Design and Mathematics, AIAA Associate Fellow.

${ }^{3}$ Senior lecturer, Department of Engineering Design and Mathematics.

${ }^{4}$ Senior lecturer, Department of Engineering Design and Mathematics.
} 


\section{Introduction}

Reduction of gas emissions $\left(\mathrm{CO}_{2}, \mathrm{NO}_{\mathrm{x}}\right)$ and noise are becoming fundamental design criteria for future aircraft, especially with the high growth rate of air travel which is estimated to double in the coming 20 years [1]. These constraints have made it necessary for engineers to pursue innovative and distributive aircraft designs that combine various groundbreaking technologies e.g. new propulsion systems, smart materials, unconventional airframe configurations and flow control devices, one of which is the use of morphing structures. Morphing structures can be optimized throughout all flight phases to obtain optimal aerodynamic performance, and morphing applied to wings is beneficial on many levels e.g. using morphing wings would improve the aerodynamic performance in off-design operating conditions by adjusting the wing surfaces in real time. Furthermore, an ideal morphing wing would effectively eliminate the gaps formed by discrete control surfaces (flaps, slats, ailerons) which would significantly streamline the wing geometry and reduce pressure drag and airframe noise. Morphing wings research as a field has been thriving over the last few years, and various studies have been conducted dealing with many aspects of morphing e.g. optimization, flight dynamics, control, and materials science [2-4]. In addition, various experiments and demonstrator concepts have been built to test the capabilities of morphing mechanisms [5-9]. Furthermore, a few papers have compiled detailed reviews of the various morphing concepts, their advantages and challenges, e.g. [1012].

The aerodynamic modelling of aircraft equipped with morphing wings has been addressed extensively in literature, the focus has been mainly on the study of the possible benefits from using this technology as a passive way to control the flow and improve the aerodynamic performance. Lyu et al. [13] conducted a shape optimization study on an adaptive morphing Trailing Edge Flap (TEF) using an in-house code to solve the Reynolds Averaged Navier-Stokes (RANS) equations with Spalart-Allmaras (SA) turbulence model. A 5\% drag reduction was observed near off-design conditions and $1 \%$ at on-design conditions. Moreover, numerical and experimental results suggested that the wing equipped with the morphing TE section exhibited superior aerodynamic performance compared with a wing equipped with a simple hinged flap. Woods et al. [14] introduced the Fish Bone Active Camber (FishBAC) concept, where they performed an aerodynamic parametric study on various morphing parameters in addition to the wind tunnel testing of the prototype [15]. Both methods demonstrated the aerodynamic superiority of a morphing TEF compared to the hinged flap, however, the experiments showed a reduction of the aerodynamic efficiency of the morphing TE flap at angles of attack closer to the stall. Abdessemed et al. [16, 17] expanded on the study of Woods et al. [14] by introducing time dependency in the parametrization method of the FishBAC [14] and analyzed the unsteady morphing of an airfoil equipped with a morphing TEF. However, only the downward deflection was considered, and the low fidelity unsteady RANS with $k \omega$-SST turbulence model was used. The study confirmed the previous steady state results e.g. [14], and suggested that the morphing motion has an influence on the flow field

Nevertheless, the use of morphing flaps as an active flow control has not thoroughly been explored as opposed to the use of oscillating, discreet hinged flaps for instance. Krzysiak et al.[18] investigated a pitching NACA 0012 airfoil with a harmonically deflecting TEF in a subsonic wind tunnel and compared the results with theoretical calculations. It was found that an increase in the maximum lift coefficient is possible when both the angle of attack of the airfoil and flap deflection increase simultaneously. Lee et al. [19] carried out a similar study where a pitching NACA 0015 airfoil with a harmonically deflected flap was experimentally tested; the study showed evidence of a great impact on the $C_{L}$ and $C_{M}$ hysteresis while the leading-edge vortex formation and detachment was found to be unaffected by the flap motion. Jones et al. $[20,21]$ investigated both experimentally and numerically the use of periodic surface morphing for flow control purposes where the suction side of an airfoil was periodically morphed at various frequencies. Results showed that the actuation frequency dominated the spectra, causing the large coherent structures to add an extra momentum and effectively reduce the separation region, thereby reducing the drag. Most recently, an experimental study was conducted by Jodin et al.[22] where the use of a piezo-actuated vibrating TE flap was investigated. It was found that a $20 \mathrm{~dB}$ power reduction of the predominant frequency could be obtained due to a substantial reduction in large scale instabilities. Likewise, a 5\% reduction in drag and 2\% increase in lift was observed at optimal combinations of frequency and amplitude. Finally, Abdessemed et al.[23] examined the effects of a harmonically morphing TEF on the acoustic spectra at a fixed actuation frequency and variable amplitudes, it was discovered that a $4 \%$ enhancement in aerodynamic efficiency was possible, in addition to the possibility of shifting the main tonal frequency to higher frequencies possibly outside human's audible range. However, an emphasis was made on the fact that the best actuation frequency/amplitude pair needs to be chosen for optimal performance improvements. Despite these recent efforts, further research work is still required to gather more data and to establish a more consistent understanding of the effects of morphing TEFs on the performance of wings. In particular, there is 
a need to investigate such effects using high fidelity CFD methods that offer greater capability for resolving unsteady flows characteristic of morphing applications.

In this paper, an unsteady computational fluid dynamics (CFD) analysis of a NACA 0012 airfoil fitted with an oscillating morphing TEF is conducted. A parametric study of the effects of the morphing frequency and amplitude on the aerodynamic performance is examined using an unsteady parametric method for the geometry deformation. The latter was implemented in a user-defined function (UDF) with appropriate smoothing techniques employed for mesh deformation. Finally, initial results comparing the downstroke oscillation to the upstroke oscillation are presented and discussed.

\section{Problem definition}

The effects of periodic forcing using a NACA 0012 airfoil fitted with a harmonically oscillating TEF on the aerodynamic performance are investigated. All test cases used an airfoil with a chord $c=0.2286 \mathrm{~m}$, a Reynold number based on the chord $\mathrm{Re}=0.62 \times 10^{6}$ and Mach number of 0.115 . The deformation of the TEF was parametrized using a modified method of the unsteady approach introduced in [17], then the method was adapted so that the entire range of motion could be achieved. The thickness distribution of the NACA 0012 wing was added to a parametric definition of the wing (Eq.1) which allows the control of the portion morphed, the amplitude of the oscillation and its frequency.Fig.1 illustrates the airfoil with the fitted TEF, and various parameters introduced in Eq.1.

$$
y_{c}=\left\{\begin{array}{c}
0 \quad, 0 \leq \bar{x}<x_{s} \\
\frac{-w_{t e} \sin (2 \pi t f)\left(\bar{x}-x_{s}\right)^{3}}{\left(1-x_{s}\right)^{3}}, \bar{x} \geq x_{s}
\end{array}\right.
$$

where $w_{t e}$ is the value of maximum deflection at the TE, $f$ is the morphing frequency and $x_{s}$ is the start location for the morphing (fixed at $75 \%$ of the chord).

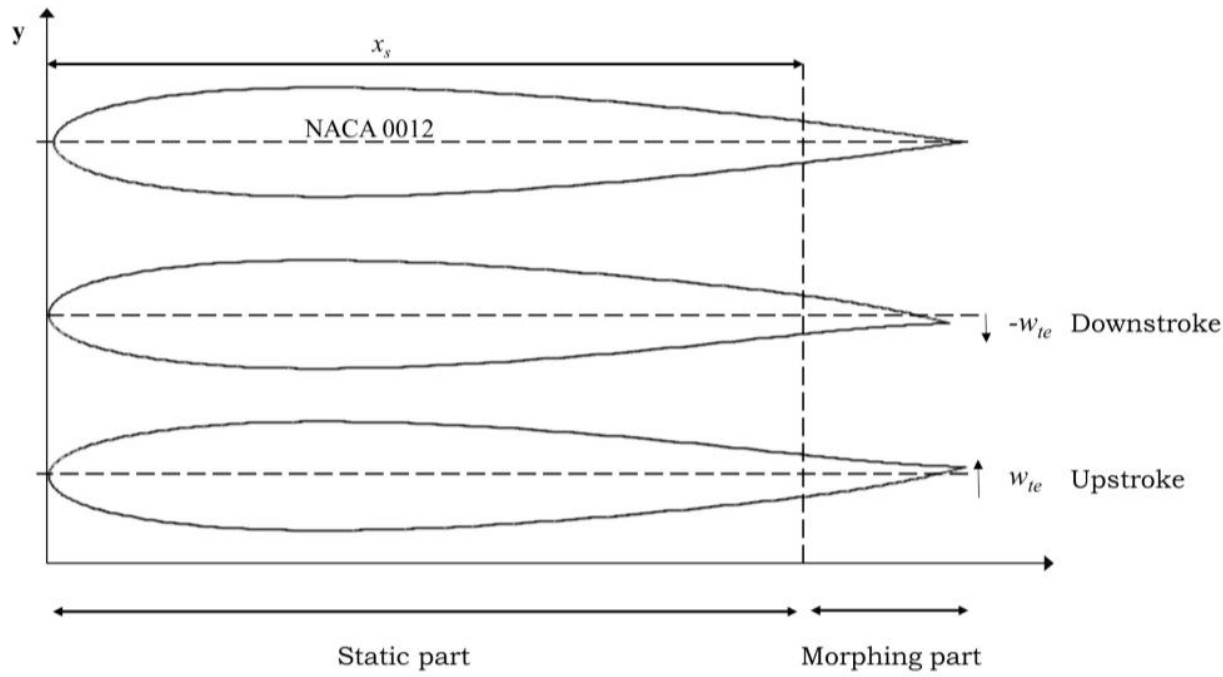

Fig.1 Illustration showing the baseline NACA 0012 with the parameters driving the morphing. 
Table. 1 Summary of the cases investigated.

\begin{tabular}{|l|c|c|c|c|c|}
\hline \multicolumn{7}{|c|}{ Fixed Amplitude $=\mathbf{0 . 0 1 \%} \boldsymbol{c}$} \\
\hline Frequencies (Hz) & 120 & 240 & 480 & 600 & 900 \\
\hline \multicolumn{7}{|c|}{ Fixed Frequency $=\mathbf{4 8 0 H z}$} \\
\hline Amplitudes (\%c) & 0 & 0.01 & 0.05 & 0.1 & 0.5 \\
\hline
\end{tabular}

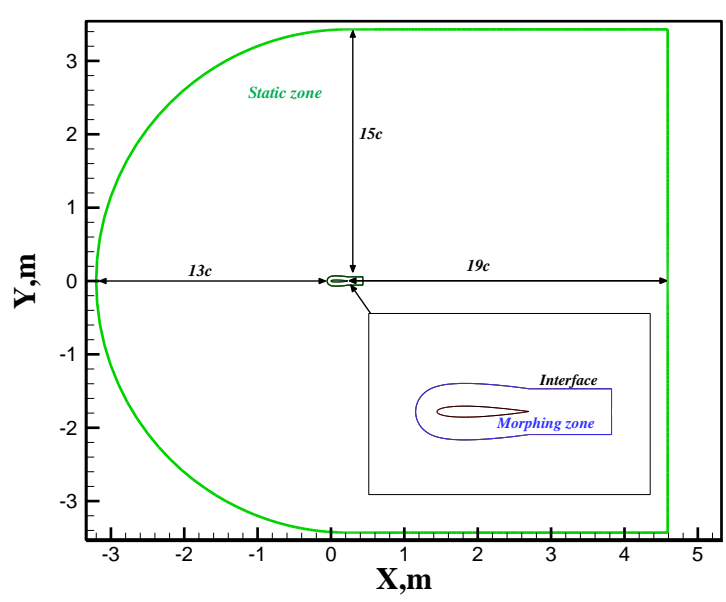

a) Illustration of various mesh zones

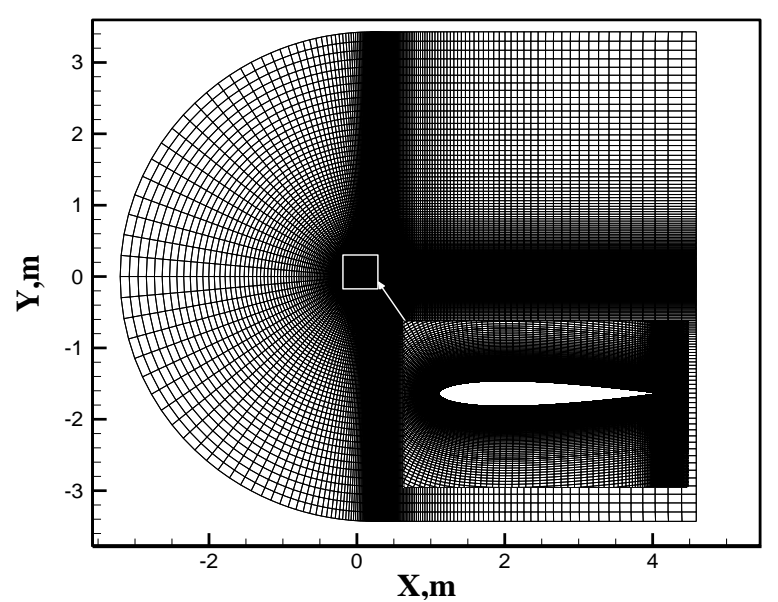

b) Mesh used for the simulations

Fig.2 Illustration showing details of the computational domain

Using Eq. 1 will make it possible to achieve a symmetrical TEF oscillation around the baseline position (between $w_{t e}$ and $-w_{t e}$ ). For an asymmetrical oscillation (downstroke from 0 to $-w_{t e}$, or upstroke from 0 to $-w_{t e}$ ) the parametric definition in Eq. 2 is used.

$$
y_{c}=\left\{\begin{array}{cc}
0 \quad, 0 \leq \bar{x}<x_{s} \\
\frac{0.5\left( \pm w_{t e}(1+\sin (2 \pi t f))\left(\bar{x}-x_{s}\right)^{3}\right.}{\left(1-x_{s}\right)^{3}}, \bar{x} \geq x_{s}
\end{array}\right.
$$

As a first step, the baseline configuration results were compared with experimental data produced by Sheldah $e t$ al. [24]. Afterwards, the parameters of amplitude and frequency were investigated for the symmetrical oscillation. A study case investigating the effects of varying the frequency for a fixed amplitude is first presented, then the frequency was fixed and the amplitude was changed. All this while maintaining an Angle of Attack $($ AoA $)=4^{\circ}$. The range of frequencies investigated was chosen relative to the shedding frequency (found to be $480 \mathrm{~Hz}$ ). The amplitude was fixed at a value equal to $0.01 \%$ of the chord similar to what is found in literature, e.g. [22]. The amplitudes were then changed while the frequency was fixed at the shedding frequency $f_{s}=480 \mathrm{~Hz}$. Finally, in order to investigate possible differences between a downstroke and an upstroke oscillation, a comparative test between the two scenarios is presented. Table 1 summarizes the cases studied.

\section{Computational Setup}

For a 2D incompressible flow, the pressure-based solver included in the commercial software Ansys Fluent [25] is used for all the subsequent unsteady calculations. The fractional step method (FSM) of the Non-Iterative TimeAdvancement Scheme (NITA) [25] was used for time advancement as it results in significant CPU savings. A least 
square cell based spatial discretization was used for all the gradients. Moreover, the momentum equation discretization employs a bounded central differencing (BCD) scheme with a second order scheme for all the remaining quantities.

Turbulence closure was provided by the Stress Blended Eddy Simulation (SBES) which is a hybrid RANS-LES turbulence model that uses a shielding function that provides significant improvements compared with older hybrid models such as the Detached Eddy simulation (DES) [26]. The RANS wall boundary layer regions are protected against influences from the LES model when the shielding functions are in use, which "shields" against early switch to the LES model that could cause a strong decline in the RANS capabilities [27].

Dynamic meshing schemes implemented in Ansys Fluent were utilized to deform the mesh. Diffusion-based smoothing was the primary smoothing method used given its suitability for structured meshes and higher quality preservation near the deformed walls [25]. In order to reduce the computational cost of using dynamic meshing, two regions were created in the mesh: a small region around the airfoil, called the morphing region, where the set of diffusion equations will be evaluated and the deformation propagating through it. The second zone, the static zone, will not be deformed thus saving CPU time. Between the two regions, an interface is created where an interpolation is performed to connect the two zones. Fig.2a illustrates the structure of the computational domain.

To capture accurately the boundary layer, the maximum near-wall first-layer grid resolution of $y^{+}<0.5$ was targeted, with an expansion ratio of 1.05 for the first 50 layers, then 1.1 for the rest of the mesh. The airfoil had 200 grid points on its surface clustered toward the leading and trailing edges, with an extra 300 points in the streamwise direction to capture the wake, resulting in a mesh size of 200k cells. A C-grid topology was used for the computational domain which extended about $19 c$ in the streamwise direction and $15 c$ in the crosswise direction. All simulations were conducted with a of time steps $\Delta t=10^{-5} \mathrm{~s}$ in order to satisfy the CFL condition of less than 1 in most of the domain. Fig2b shows the mesh used for the simulations.

\section{Results and discussion}

\section{A. Static baseline NACA 0012 results}

In this section the flow around the baseline (unmorphed) airfoil is investigated using the methedology decribed in the previous section. Results were compared with those from a wind tunnel test [24] for the same airfoil at a Reynolds number close to the one used in this study $\left(\sim \mathrm{Re}=0.7 \times 10^{6}\right)$. Figure 3 shows the numerical and experimental results for both $C_{L}$ and $C_{D}$ for a range of AoA up to $8^{\circ}$. Both lift and drag predictions agree well with the test data.

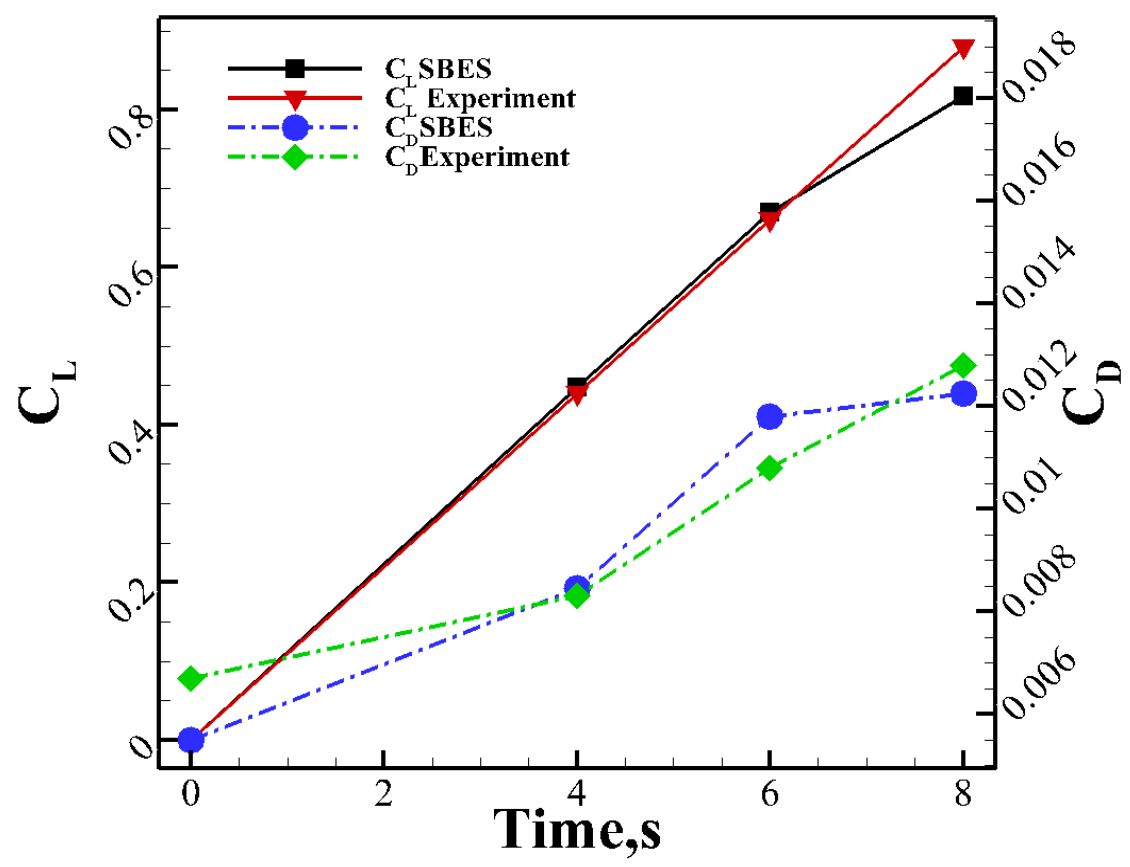

Fig.3 Results for $C_{L}$ and $C_{D}$ obtained from SBES simulation compared with experimental results for the NACA 0012 airfoil from Sheldah et al. [24]. 
The maximum discprency noted for $C_{L}$ was about $8 \%$ at AoA of $8^{\circ}$, whereas the $C_{D}$ discripency was smaller in this range of AoA (about 5\% diffrence between the test and the simulation). Moreover, for the $4^{\circ}$ AoA that we will be investigated in subsequent sections, the discrepency is less than $2 \%$ for both $C_{L}$ and $C_{D}$.

Figure 4 illustrates the lift and drag coefficients results obtained for the NACA 0012 at AoA $4^{\circ}$, this angle was chosen to replicate the study performed in [23] and explore a larger range of frequencies and amplitudes. Figure 4a and $4 \mathrm{~b}$ illustrate the instantaneous results for $C_{L}$ and $C_{D}$ taken for a period of $0.84 s$, which is approximately 4 times the Flow-Through time (TFT). A continuous vortex shedding is observed from the oscillations about the mean values. In order to have extra insight into the flow characteristics, a Fast Fourier Transform (FFT) is performed on the $C_{L}$ signal, Fig. 4c shows the resulting Power Spectral Density (PSD). A clear peak is observed from the FFT which is associated with the laminar boundary layer-vortex shedding (LBL-VS) $[28,29]$ frequency $f_{s}$ which is determined to be $480 \mathrm{~Hz}$, equivalent to a Strouhal number $S t=0.32$ (using the airfoil thickness as reference length). This value of $S t$ is expected based on the Reynolds and Strouhal number relation for bluff bodies. This frequency is used in the subsequent section as a reference for the morphing frequencies used for the harmonic forcing. From Fig. 4, one also notices the appearance of what seems to be two sub-harmonics at $213 \mathrm{~Hz}$ and $266 \mathrm{~Hz}$. High frequency peaks also appear in the spectra.

\section{B. Harmonically morphing TEF results}

As mentioned in the problem definition, parametric studies were performed in order to explore the effects that changing frequencies or amplitude has on the efficiency of harmonically morphing TEFs as active flow control devices.

\section{Effect of frequency variation}

Figure 5 shows the PSD for all the frequencies studied compared with the baseline unactuted airfoil. The main diffrence shared by all the cases comapred with the unactuted case is that the spectral density associated with the shedding frequency is significally lower at all the actuated cases, the $600 \mathrm{~Hz}$ seems to produce almost a complete reduction in the predominant frequency peak captured in the unactuted case. This behaviour could be due to possible energy transfer to the larger coherant structures by means of the periodic forcing. Similar effects were observed by
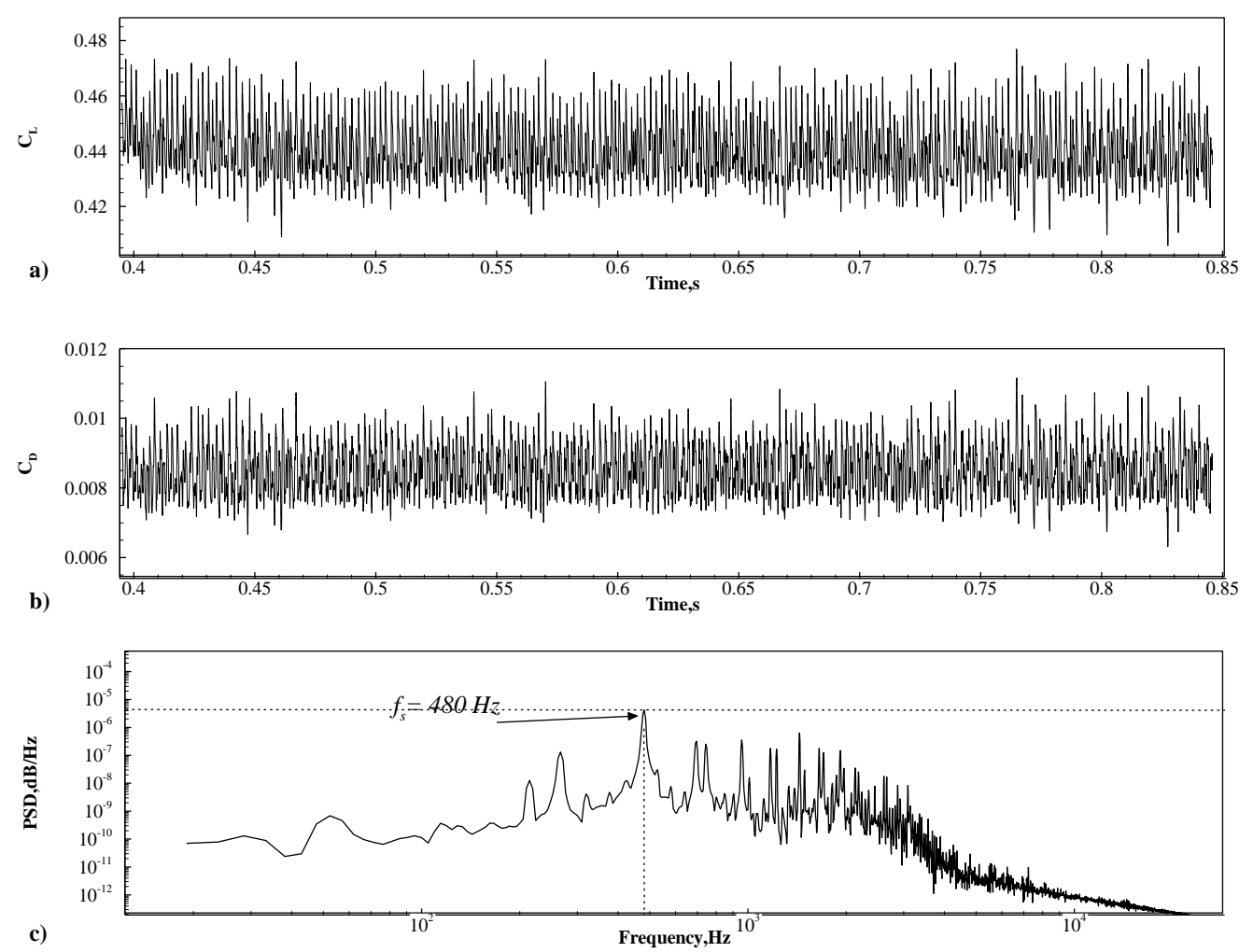

Fig.4 Time history of $C_{L}(a) C_{D}(b)$ and the spectral density of $C_{L}(c)$ for NACA 0012 at $\mathrm{AoA}=4^{\circ}$ demonstrating the shedding frequency $f_{s}$. 
Jones et al. [20,21] where the actuation was found to cause a locked-on phenomenon causing the actuation frequency to dominate the spectra. This reduction could be translated into significant noise level reductions, yet the levels of PSD observed for the current amplitudes/ frequencies seems to be weaker than what is observed in literature. Such observation gives indications that the fixed amplitude $(0.01 \%)$ is not large enough to generate LCS large enough to cause a significant disruption in the wake. This is confirmed by Fig.6 presenting the turbulence kinetic energy (TKE) contours for all the frequencies explored, there is no significant change in the flow structures between all the cases. One main difference that could be observed is that for the $900 \mathrm{~Hz}$ the actuation seems to affect wake's flow structure through what seems to be a delayed breakdown of the large vortices by breaking down the large vortex structures in the wake which could affect the drag and lift.

Figure 7 displays the change in the average value of $C_{D}, C_{L}$ and the aerodynamic efficiency $\left(C_{L} / C_{D}\right)$. For all the cases, the actuations appear to increase slightly the drag (up to $0.2 \%$ when actuating at the vortex shedding frequency) the same trend is observed when it comes to $C_{L}$ where most actuation frequency had a negative impact on the lift. The exception was the $900 \mathrm{~Hz}$ frequency as a $0.5 \%$ increase in lift was obtained, this could be linked to the relatively stronger effect this actuation frequency had on the wake. Finally, Fig.7c illustrates the change in the aerodynamic efficiency, which confirmed that, the $900 \mathrm{~Hz}$ produces the only increase in efficiency observed (about $0.4 \%$ ) whereas the remaining actuating frequency reduced the efficiency. One plausible explanation of the lack of significant effects
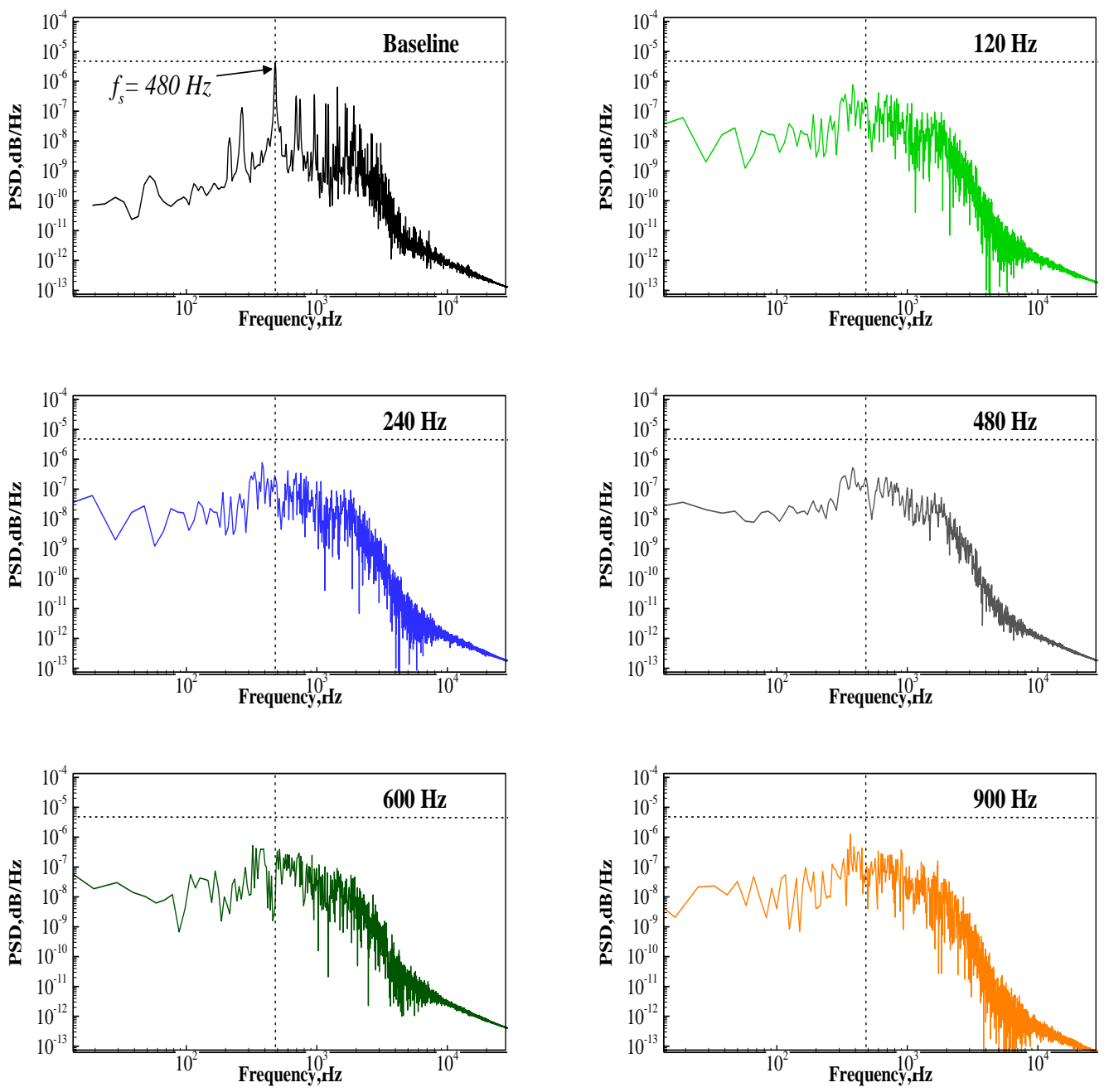

Fig.5 Power spectral density of $C_{L}$ for all the frequencies studied compared with the baseline results at $\mathrm{AoA}=4^{\circ}$ and $w_{t e}=0.01 \%$. 
on the performance is that the amplitude of morphing used is not large enough. The next section shows the effects of larger amplitudes for a fixed morphing frequency.
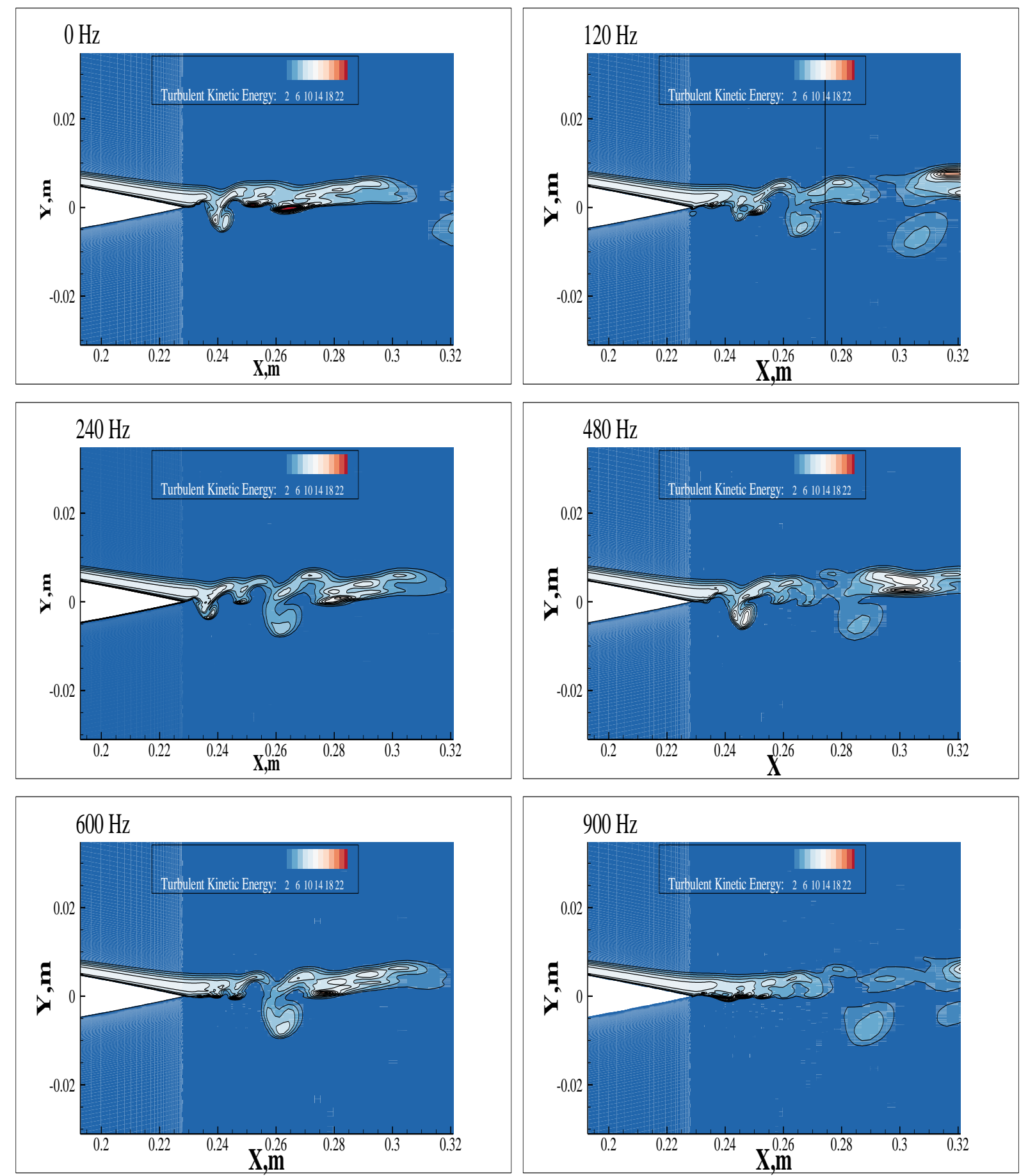

Fig.6 Turbulence Kinetic energy contours for all the frequencies studied compared with the baseline results (0 $\mathrm{Hz}$ ) at $\mathrm{AoA}=4^{\circ}$ and $w_{t e}=0.01 \%$. 


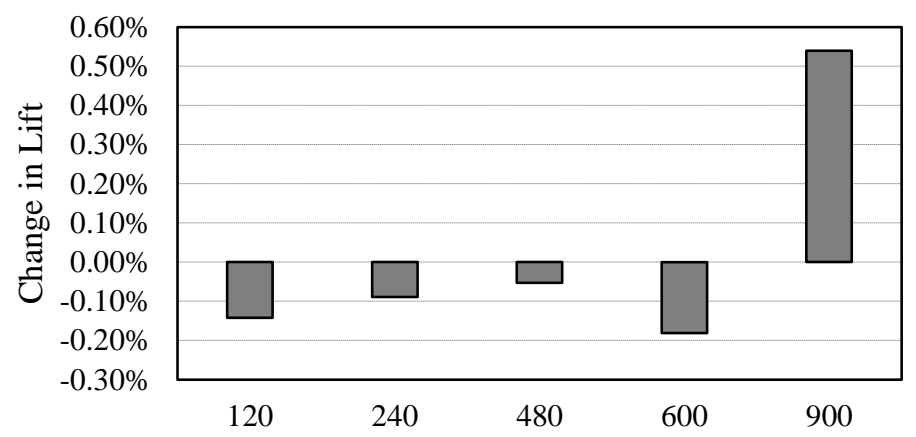

a) Change in Lift Coefficient

Frequency, $\mathrm{Hz}$

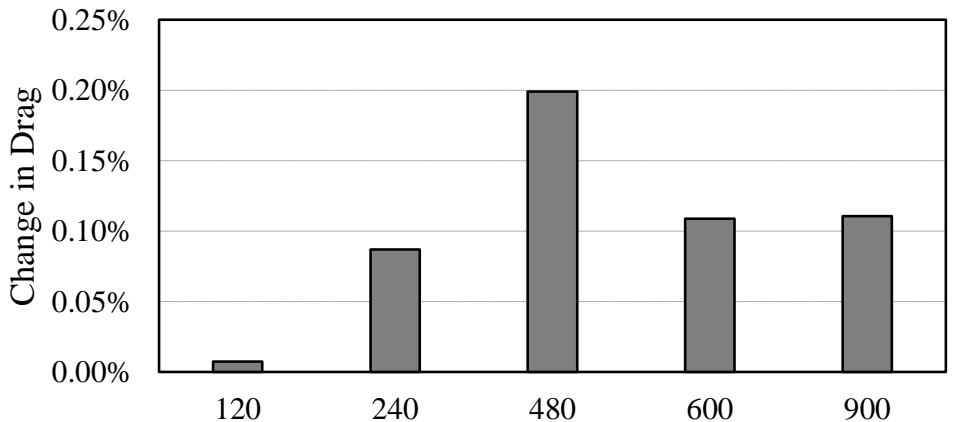

Frequency, $\mathrm{Hz}$

b) Change in Drag Coefficient.

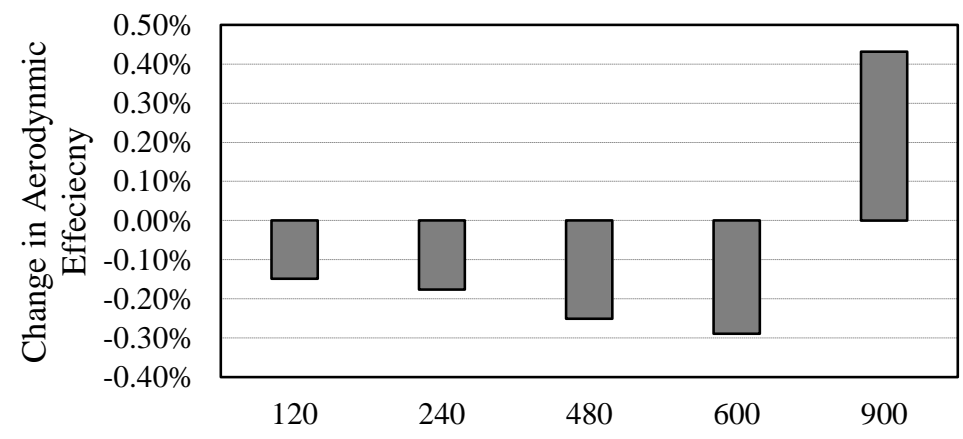

Frequency,Hz

c) Change in Aerodynamic efficiency.

Fig.7 The \% change relative to the baseline in lift, drag and aerodynamic efficiency induced by the harmonic forcing. 

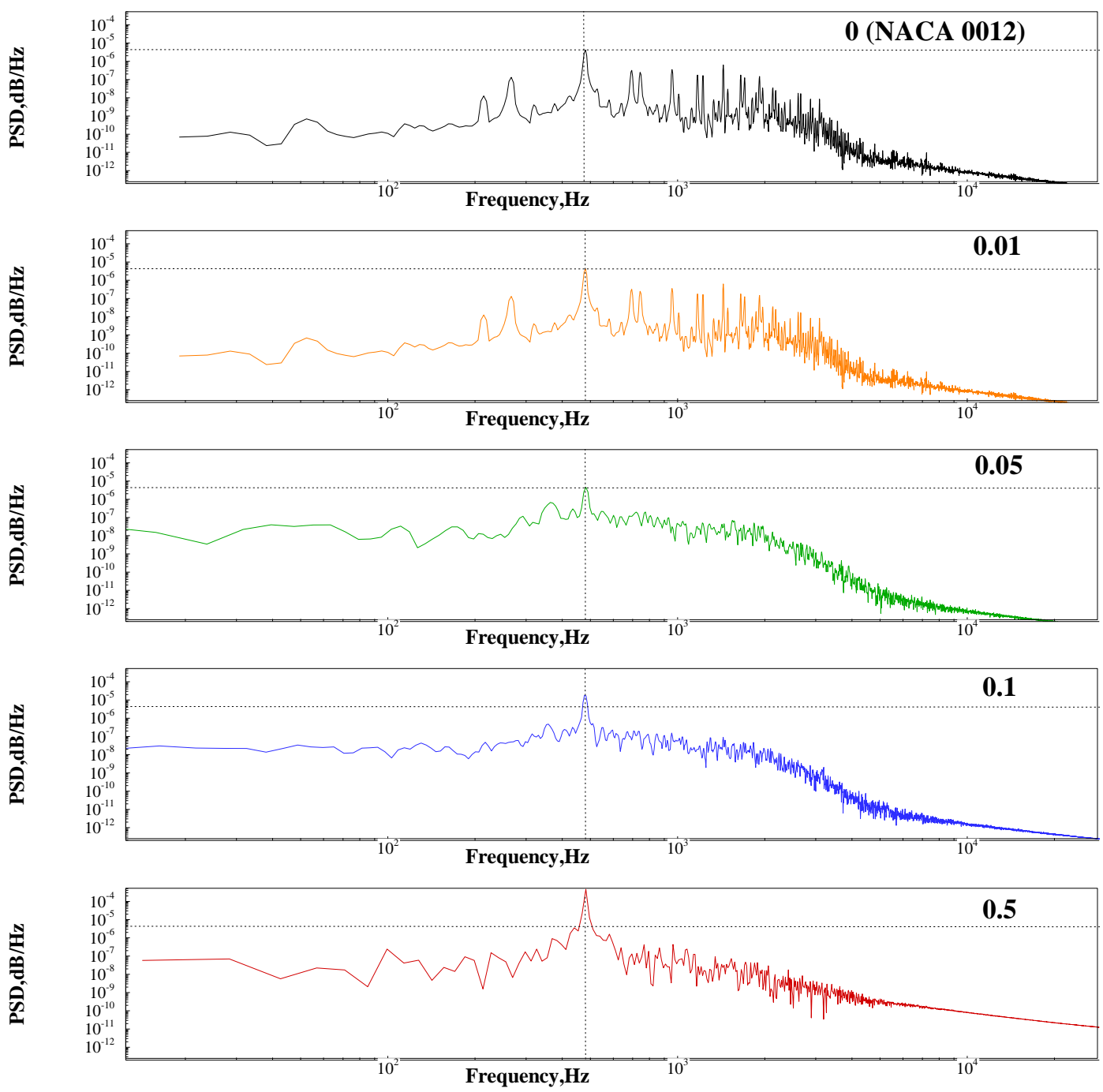

Fig.8 Power spectral density of $C_{L}$ for all the amplitudes studied compared with the baseline results at $\mathrm{AoA}=4^{\circ}$ and $f=480 \mathrm{~Hz}$.

\section{Effect of amplitude variation}

Figure 8 presents the spectral density analysis performed on the $C_{L}$ time history signal for all the morphing amplitudes investigated. Results confirm once more that that actuating frequency dominates the spectra keeping the dominant frequency at $480 \mathrm{~Hz}$ which is the frequency used for forcing. Nonetheless, the power density levels seem to only be affected at higher amplitudes starting from $0.1 \%$ where the PSD level seems to increase with higher amplitudes of morphing, this could be due to the fact that a higher amplitude induces more local camber change which might enhance the size of the local separation. Yet these higher PSD levels are associated with increased noise levels. Another effect possibly induced by morphing is that the high frequency harmonics appearing in the baseline and the $0.01 \%$ case do not appear in the rest of the cases. Such effects might be explained by the energy transfer to the larger structures produced by larger amplitudes concentrating energy at a specific frequency. Figure 9 qualitatively illustrates the differences and similarities between various actuating amplitudes. As expected from the spectral analysis, the differences are subtle or inexistent for the first two cases. However, for the $0.1 \%$ and $0.5 \%$ case, the large structures within the wake seem to be discretized into slightly smaller ones, with a more accentuated effect in the near wake for the $0.5 \%$ case, this again could have effects on the aerodynamic performance. 

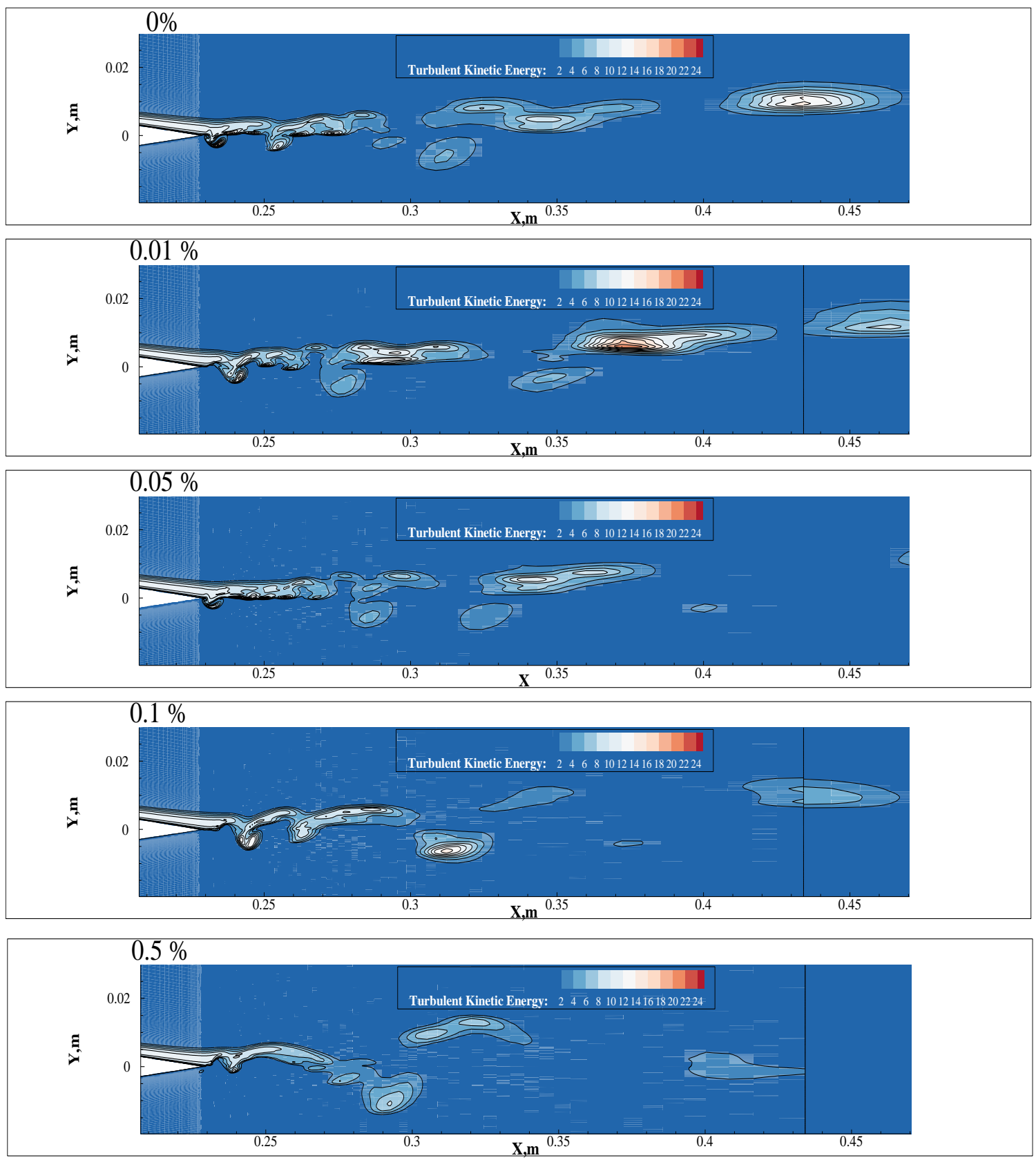

Fig.9 Turbulence Kinetic energy contours for all the amplitudes studied compared with the baseline results $(0 \%)$ at $\mathrm{AoA}=4^{\circ}$ and $f=480 \mathrm{~Hz}$. 


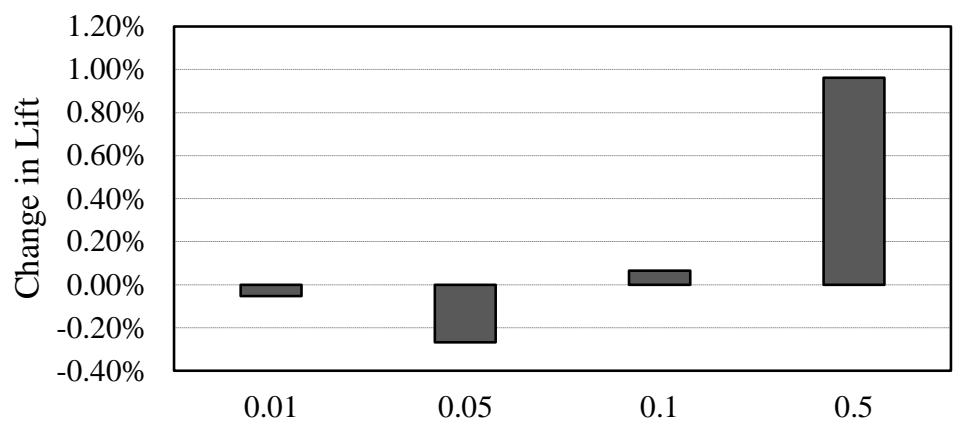

Amplitude.\%c

a) Change in Lift Coefficient.

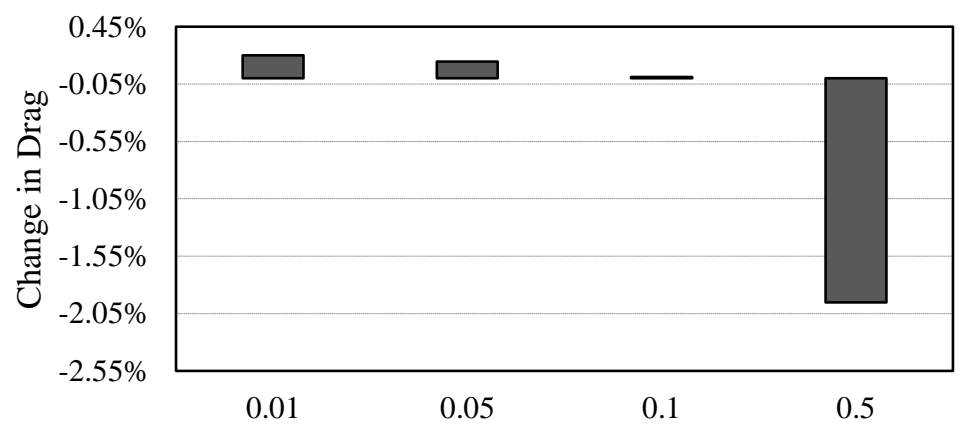

Amplitude.\%c

b) Change in Drag Coefficient.

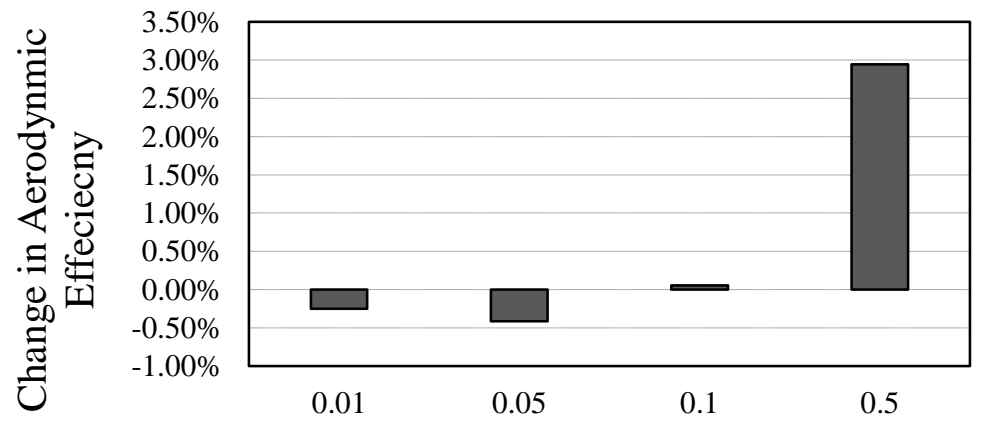

Amplitude, $\% c$

c) Change in Aerodynamic efficiency.

Fig.10 Change relative to the baseline in lift, drag and aerodynamic efficiency induced by the harmonic forcing for a fixed frequency $(480 \mathrm{~Hz})$ and variable amplitudes. 
Figure 10 shows the average value of the change in $C_{D}, C_{L}$ and the aerodynamic efficiency for each amplitude studied calculated relative to the unactuated NACA 0012. A clear trend appears in the results where smaller amplitudes (0.01 and 0.05) have negative impact on the aerodynamic efficiency with the drag experiencing a slight increase and the lift decreasing as low as $-0.3 \%$, however this trend is reversed at amplitude $0.1 \%$ where the lift experiences a slight increase for similar levels of drag. The highest increase in the aerodynamic efficiency was observed for $0.5 \%$, up to $3 \%$ increase of efficiency was obtained using this amplitude which is caused by the simultaneous decrease in drag $(2 \%)$ and increase in lift $(1 \%)$. This decrease in drag could have originated from the fact that the wake width was affected by the shrinking of the large coherent structures in the near wake which translated in a decrease in pressure drag.

\section{Downstroke vs upstroke}

A test case comparing between the use of asymmetrical oscillation (only an upstroke or a downstroke) was performed to investigate possible impact on the aerodynamic performance. Both the frequency and amplitude $(f=480$ $\mathrm{Hz}, w_{t e}=0.05 \% c$ ). initial results showed that the impact on the forces is different depending on which stroke is used, for the upstroke both lift and drag decreased leading to a $-0.3 \%$ decrease in the aerodynamic efficiency. However, the downstroke showed better performance with a $2 \%$ increase in lift along with a $1 \%$ increase in drag, which translated by a $1 \%$ increase in the aerodynamic efficiency. This give an indication that the way the TEF is oscillated could have an impact on the efficiency of the flow control mechanism.

\section{Conclusion and Future Work}

The effects of changing the frequency and amplitude of a harmonically oscillating TEF were investigated and the results compared with the baseline unmorphed configuration at a $\mathrm{Re}=0.62 \times 10^{6}$ for a $4^{\circ}$ AoA. For a given amplitude a set of frequencies lower or higher than the shedding frequency were examined, along with a range of amplitudes for a fixed frequency.

Spectral analysis confirmed findings from previous studies namely that the morphing frequencies dominate the spectra, with a significant reduction in the predominant frequency peak which is associated with the LBL-VS. It was also found that for the amplitude used $(0.01 \%$ of the chord) only a frequency higher than the shedding frequency produced enhanced aerodynamic efficiency due to the breakdown of large coherent structures in the near wake.

The study of various amplitudes concluded that higher amplitudes produced better efficiency for the studied frequency. By using an amplitude of $0.5 \%$ of the chord a $2 \%$ decrease in the total drag was obtained producing up to $3 \%$ increase in the aerodynamic efficiency.

Moreover, a preliminary study into the effect of using only the downstroke or upstroke oscillation showed that the two scenarios behave differently, an increase of $1 \%$ in the aerodynamic efficiency was obtained using the downward oscillation whereas the upstroke resulted in a slight efficiency loss.

Finally, this study confirmed that in order to obtain optimal reductions in drag, increases in lift and overall improvement in the aerodynamic efficiency it is important to pair the right frequency and amplitude.

In the future, an in-depth investigation of the entire design space is needed in order to have a complete image, and possibly a correlation, of the optimal frequency, amplitude and size of the flap needed to obtain best performance gains using the harmonic forcing by a morphing TEF. Moreover, an investigation of higher angles of attack is primordial as periodic forcing is expected to have greater effects near stall. Additionally, a deeper investigation of the effect of downstroke or upstroke oscillation needs to be performed to gain an understanding of possible differences.

Lastly, the complex flow phenomena occurring undoubtedly need full 3D CFD analysis with LES model or Direct Numerical Simulation (DNS) to unfold fully the complexities in the flow.

\section{ACKNOWLEDGEMENTS}

The first author gratefully acknowledges the studentship funding received from the Engineering Modelling and Simulation Research Group, University of the West of England, Bristol, UK. 


\section{References}

[1] IATA, "IATA forecasts passenger demand to double over 20 Years," Press Release, no. 59, 2016.

[2] Prock, B. C., Weisshaar, T. A., and Crossley, W. A "Morphing Airfoil Shape Change Optimization With Minimum Actuator Energy As An Objective," 9th AIAA/ISSMO Symposium on Multidisciplinary Analysis and Optimization, Multidisciplinary Analysis Optimization Conferences, pp. 4-6, 2002.doi : 10.2514/6.20025401.

[3] Urnes, J., Nguyen, N., Ippolito, C., Totah, J., Trinh, K., and Ting, E "A Mission-Adaptive Variable Camber Flap Control System to Optimize High Lift and Cruise Lift-to-Drag Ratios of Future N + 3 Transport Aircraft," 51st AIAA Aerospace Sciences Meeting including the New Horizons Forum and Aerospace Exposition. 7-10 January, Grapevine,. January, pp. 1-24, 2013.doi: 10.2514/6.2013-214.

[4] Koreanschi, A. et al., "Optimization and design of an aircraft's morphing wing-tip demonstrator for drag reduction at low speed, Part I - Aerodynamic optimization using genetic, bee colony and gradient descent algorithms," Chinese Journal of Aeronautics, vol. 30, no. 1, pp. 149-163, 2017.doi: 10.1016/j.cja.2016.12.013.

[5] Beaverstock, C.S., Woods, B.K.S., Fincham, J.H.S.M. and Friswell, M.I., "Performance Comparison between Optimised Camber and Span for a Morphing Wing," Aerospace, vol. 2, no. 3, pp. 524-554, 2015.doi: 10.3390/aerospace2030524.

[6] Chen, Y., Yin, W., Liu, Y. and Leng, J., "Structural design and analysis of morphing skin embedded with pneumatic muscle fibers,"Smart Materials and Structures., vol. 20, no. 8, p. 85033, 2011.doi: 10.1088/0964$1726 / 20 / 8 / 085033$.

[7] Woods, B. K. S., Bilgen, O. and Friswell, M. I."Wind tunnel testing of the fish bone active camber morphing concept," Journal of Intelligent Material Systems and Structures.vol. 22, no. 7, pp. 364-375, 2013. doi: 10.1177/1045389X14521700.

[8] Takahashi, H., Yokozeki, T. and Hirano, Y., "Development of variable camber wing with morphing leading and trailing sections using corrugated structures," Journal of Intelligent Material Systems and Structures, vol. 27, no. 20, pp. 2827-2836, Apr. 2016.doi: 10.1177/1045389X16642298.

[9] Gabor, O.Ş., Koreanschi, A., Botez, R.M., Mamou, M. and Mebarki, Y., "Numerical simulation and wind tunnel tests investigation and validation of a morphing wing-tip demonstrator aerodynamic performance," Aerospace Science Technology., vol. 53, pp. 136-153, 2016.doi: 10.1016/j.ast.2016.03.014.

[10] Barbarino, S., Bilgen, O., Ajaj, R. M., Friswell, M. I., and Inman, D. J. "A Review of Morphing Aircraft," Journal of intelligent material systems and structures, vol. 22, no. 9, pp. 823-877, 2011. doi: 10.1177/1045389X11414084.

[11] Lachenal, X., Daynes, S. and Weaver, P.M."Review of morphing concepts and materials for wind turbine blade applications," Wind Energy, vol. 16, no. 2, pp. 283-307, Mar. 2013. .doi: 10.1002/we.531.

[12] Weisshaar, T. A. "Morphing aircraft technology-new shapes for aircraft design Multifunctional Structures/Integration of Sensors and Antennas." In Meeting Proceedings RTOMP-AVT-141, Overview 1. Neuilly-sur-Seine, France: RTO, p. O1. 2006.

[13] Lyu, Z., and Martins, J. R. "Aerodynamic Shape Optimization of an Adaptive Morphing Trailing-Edge Wing," Journal of Aircraft,AIAA., vol. 52, no. 6, pp. 1951-1970, 2015.doi: 10.2514/1.C033116.

[14] Woods, B. K., Fincham, J. H., \& Friswell, M. I. "Aerodynamic Modelling of the Fish Bone Active Camber Morphing Concept Aerodynamic Modelling of the Fish Bone Active Camber Morphing Concept," Journal of Intelligent Material Systems and Structures. SAGE Publications, 25(7), pp. 772-785. July, 2014. doi: $10.1177 / 1045389$ X14521700

[15] Woods, B. K. S., Bilgen, O. and Friswell, M. I. "Wind tunnel testing of the fish bone active camber morphing concept," Journal of Intelligent Material Systems and Structures. SAGE Publications, 25(7), pp. 772-785. 2014.doi: 10.1177/1045389X14521700.

[16] Abdessemed,C., Yao,Y., Bouferrouk,A., and Narayan,P. "Unsteady parametrization of a morphing wing design for improved aerodynamic performance," 52nd 3AF International Conference on Applied Aerodynamics, Lyon, France, 27-29 March 2017. Available from: http://eprints.uwe.ac.uk/31078.

[17] Abdessemed, C., Yao, Y., Bouferrouk, A., \& Narayan, P. "Morphing airfoils unsteady flow analysis using dynamic meshing," International Journal of Numerical Methods for Heat \& Fluid Flow, vol. 23, no. 5, 2017.doi: 10.1108/HFF-06-2017-0261

[18] Krzysiak, A. and Narkiewicz, J., "Aerodynamic Loads on Airfoil with Trailing-Edge," Journal of Aircraft, Vol. 43, No. 2 2006, pp. 407-418.doi: 10.2514/1.15597. 
[19] Lee, T. and Gerontakos, P., "Unsteady Airfoil with Dynamic Leading- and Trailing-Edge Flaps," Journal of Aircraft, 46(3), p.1076. , 2009. doi: 10.2514/1.42431.

[20] Jones, G., Santer, M., Debiasi, M., \& Papadakis, G. "Control of flow separation around an airfoil at low Reynolds numbers using periodic surface morphing," Journal of Fluids and Structures, vol. 76, pp. 536-557, 2018. doi: 10.1016/j.jfluidstructs.2017.11.008.

[21] Jones, G., Santer, M., \& Papadakis, G. "Control of low Reynolds number flow around an airfoil using periodic surface morphing: A numerical study" Journal of Fluids and Structures, vol. 76, pp. 95-115, 2018. doi: 10.1016/j.jfluidstructs.2017.09.009.

[22] Jodin, G., Motta, V., Scheller, J., Duhayon, E., Döll, C., Rouchon, J. F., \& Braza, M. "Dynamics of a hybrid morphing wing with active open loop vibrating trailing edge by time-resolved PIV and force measures," Journal of Fluids and Structures, vol. 74, pp. 263-290, 2017. doi: 10.1016/j.jfluidstructs.2017.06.015.

[23] Abdessemed, C., Yao, Y., Bouferrouk, A., \& Narayan, P "Aeroacoustic Investigation of a Harmonically Morphing Trailing Edge Flap," 53rd 3AF International Conference on Applied Aerodynamics, Salon de Provence, France, 26 - 28 March 2018. Available from: http://eprints.uwe.ac.uk/35578

[24] Sheldahl, R. E., \& Klimas, P. C. "Aerodynamic characteristics of seven symmetrical airfoil sections through 180-degree angle of attack for use in aerodynamic analysis of vertical axis wind turbines," Sandia National Labs., Albuquerque, NM (USA), 1981.

[25] ANSYS, (2018) "Release 18.2, Help System." ANSYS Fluent Theory Guide. ANSYS, Inc, 2018.

[26] Spalart, P. R. "Detached-eddy simulation," Annual review of fluid mechanics 41 (2009): 181-202. 2009.doi: 10.1146/annurev.fluid.010908.165130.

[27] Spalart, P.R., Deck, S., Shur, M.L., Squires, K.D., Strelets, M.K. and Travin, A., “A new version of detachededdy simulation, resistant to ambiguous grid densities," Theoretical and computational fluid dynamics, 20(3), pp.181-195. , 2006.doi: 10.1007/s00162-006-0015-0.

[28] Yarusevych, S., Sullivan, P. E., \& Kawall, J. G. "Coherent structures in an airfoil boundary layer and wake at low Reynolds numbers," Physics of Fluids, vol. 18, no. 4, p. 44101, 2006.doi: 10.1063/1.2187069.

[29] Brooks, T. F., Pope, D. S., \& Marcolini, M. A. , “Airfoil self-noise and prediction,” NASA-RP-1218 , 1989. 